\title{
Comportamiento de la referencia a trasplante renal en el Instituto Mexicano del Seguro Social
}

\author{
Behavior of the reference to renal transplantation \\ in the Mexican Social Security Institute
}

\begin{abstract}
Antonio Méndez-Durán*
* Nefrólogo de Adultos. Especialista en Hipertensión Arterial. Especialista en Diabetes. Coordinador de Programas Médicos. Coordinación de Planeación de Infraestructura Médica. Dirección de Prestaciones Médicas del Instituto Mexicano del Seguro Social. Nivel Central. Ciudad de México, México.
\end{abstract}

\section{RESUMEN}

Introducción: Las terapias sustitutivas de la función renal constituyen un soporte de vida; sin embargo, son tratamientos temporales hasta realizar el trasplante renal (TR); mientras tanto, las complicaciones están presentes en el deterioro de la calidad y reducción de la expectativa de vida. El TR rehabilita y da la posibilidad de realizar una vida normal, reintegración familiar, social y laboral. La referencia a TR día a día se torna más frecuente y conocer el comportamiento permitirá implementar estrategias de mejora. Material y métodos: Estudio retrospectivo y transversal realizado de 2014 a 2019 . Incluye datos de referencias a TR generadas por los hospitales generales y regionales del IMSS de sujetos de hasta 65 años de edad. Se identifica localidad de la referencia, género, edad, causa de insuficiencia renal crónica (IRC) y modalidad de terapia sustitutiva. Resultados: Se refirieron 19,979 pacientes; 12,386 fueron masculinos (62\%) y 7,593 femeninos (38\%), con edades de 1 a 65 años, el grupo de 20 a 29 años fue el más prevalente (30\%). El mayor número de referencias recibió diálisis peritoneal 9,166 (46\%), hemodiálisis 8,981 (45\%), y 1,832 (9\%) de prediálisis. La referencia incrementó $8.7 \%$ promedio anual en todas las modalidades dialíticas. Conclusiones: La referencia presenta un incremento continuo y los pacientes de prediálisis cada año son referidos en mayor número. La demanda del trasplante renal supera las necesidades de la institución y ofrece una gran área de oportunidad para favorecer a un mayor número de pacientes.

Palabras clave: Insuficiencia renal crónica, donador vivo, receptor, referencia, trasplante renal.

\section{ABSTRACT}

Introduction: The substitute therapies of the renal function constitute a support of life, nevertheless they are temporary treatments until realizing renal transplantation $(T R)$, meanwhile the complications are present the deterioration of the quality and reduction of the life expectancy. The TR rehabilitates, gives the possibility of a normal life, family, social and labor reintegration. The reference to TR day by day becomes more frequent and knowing the behavior will allow to implement improvement strategies. Material and methods: Retrospective and cross-sectional study conducted from 2014 to 2019, includes data on references to $T R$ generated by the general and regional hospitals of the IMSS of subjects up to 65 years of age. Reference location is identified, gender, age, cause of chronic renal failure (CRF), modality of replacement therapy. Results: 19,979 patients were referred, 12,386 were male (62\%) and 7,593 female (38\%), aged 1 to 65 years, the group of 20 to 29 years was the most prevalent (30\%). The highest number of references received peritoneal dialysis 9,166 (46\%), hemodialysis 8,981 (45\%), and 1,832 (9\%) pre-dialysis. The reference increased $8.7 \%$ annual average in all the dialysis modalities. Conclusions: The reference presents a continuous increase and pre-dialysis patients each year are referred in greater numbers. The demand for renal transplantation exceeds the needs of the institution and offers a large area of opportunity to favor a greater number of patients.

Keywords: Chronic renal failure, living donor, recipient, reference, renal transplant. 


\section{INTRODUCCIÓN}

En México, el proceso de donación renal es complejo, los procesos administrativos no estandarizados y más aún los médicos, tampoco, lo cual demora el proceso de estudio de los pacientes que requieren de un trasplante renal (TR). De acuerdo con el artículo 333 de la Ley General de Salud, para realizar un trasplante de donador vivo deben cumplirse ciertos requisitos por parte del donante y protocolos establecidos por las instancias normativas, bajo el deseo genuino de donar. ${ }^{1}$ Debido a la gran penetración de los servicios de salud, el Instituto Mexicano del Seguro Social actualmente realiza el mayor número de trasplantes en el país, con resultados satisfactorios. ${ }^{2}$ Sin embargo, la incidencia y prevalencia de la enfermedad renal crónica (ERC) incrementadas rebasan la capacidad resolutiva de la cirugía de $\mathrm{TR}^{3}{ }^{3}$ El número de trasplantes en cada medición realizada resulta en un incremento; no obstante, de manera similar ocurre con el registro de pacientes con ERC que requieren de TR. ${ }^{4}$ Por ello, es necesaria la implementación de un programa de salud renal que, a través de estrategias preventivas y de intervención temprana, permita incidir en un corto, mediano y largo plazo en la reducción del número de enfermos renales, en los ingresos a terapias dialíticas y en el requerimiento de un trasplante renal. Posterior a seis años del registro de Referencia a Trasplante Renal, es necesario identificar las principales características de los potenciales receptores de riñón en el Instituto Mexicano del Seguro Social que permitan identificar áreas de mejora.

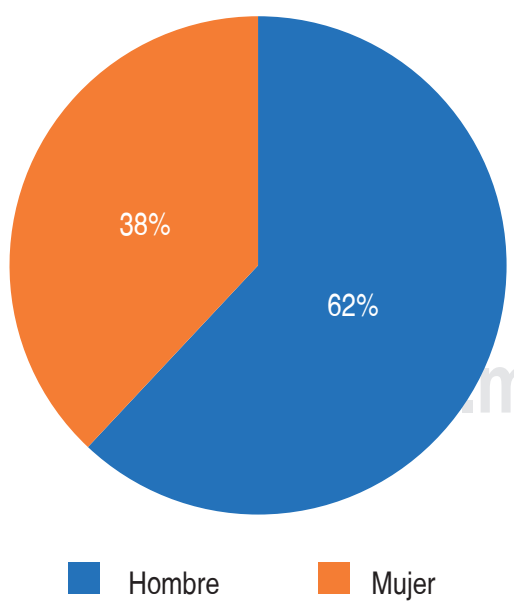

Figura 1: Distribución por género ( $\mathrm{N}=19,979)$. Relación masculino:femenino 1.6:1.
Tabla 1: Distribución de pacientes referidos por grupos de edad.

\begin{tabular}{cr}
\hline Grupo de edad & $\%$ \\
\hline 1 a 9 & 0.1 \\
10 a 19 & 4.0 \\
20 a 29 & 30.0 \\
30 a 39 & 20.0 \\
40 a 49 & 18.0 \\
50 a 59 & 18.0 \\
60 a 65 & 10.0 \\
Total & 100.0 \\
\hline
\end{tabular}

\section{MATERIAL Y MÉTODOS}

Estudio retrospectivo y transversal de seis años de seguimiento (enero 2014 a diciembre 2019), los datos se obtuvieron de los registros de referencia de pacientes a protocolo de estudio para trasplante renal generados por los hospitales generales y regionales del IMSS. La información fue revisada y concentrada por estado y hospital, se identifica en los pacientes el género, edad, causa de la insuficiencia renal crónica (IRC), modalidad de terapia sustitutiva y tipo de donador (vivo o cadavérico). El número de pacientes en terapias dialíticas fue tomado del Censo de Insuficiencia Renal Crónica de la misma institución y el número de pacientes trasplantados del informe anual proporcionado por el Centro Nacional de Trasplantes (CENATRA). De los pacientes en terapias dialíticas, referidos y trasplantados se obtienen porcentajes.

\section{RESULTADOS}

Se refirieron 19,979 potenciales receptores; 2,479 en 2014; 3,005 en 2015; 3,036 en 2016; 2,722 en 2017; 4,391 en 2018 y 4,346 en 2019; un incremento anual de $8.7 \%$. Según el género, 12,386 fueron masculinos $(62 \%)$ y 7,593 femeninos (38\%), con una relación 1.6:1.0 (Figura 1). Las principales causas de insuficiencia renal crónica fueron: hipertensión arterial 51\%, glomerulopatías crónicas de etiología no determinada $23 \%$, nefropatía tubulointersticial $21 \%$, causas congénitas $4 \%$ y otros $1 \%$. Los pacientes referidos presentaron edad desde un año hasta 65, el grupo de 20 a 29 años fue el más prevalente (30\%) (Tabla 1). Según la modalidad dialítica, el mayor número de enfermos referidos recibió diálisis peritoneal (46\%), hemodiálisis $45 \%$, y se encontraron en prediálisis $9 \%$ (Figura 2). Setenta y ocho por ciento de los pacientes fueron referidos a estudio de donación cadavérica. La delegación 


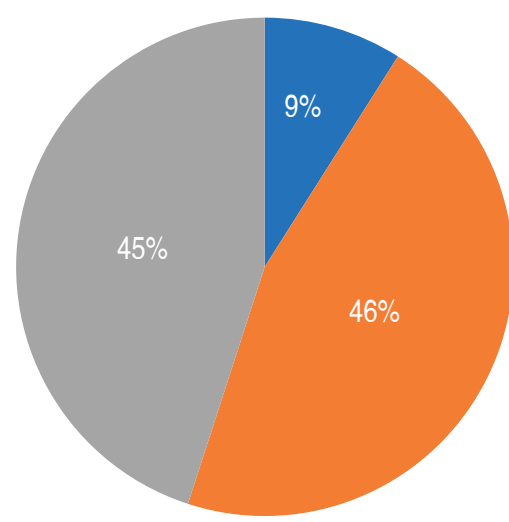

Prediálisis

Diálisis peritoneal

Hemodiálisis

Figura 2: Distribución de la referencia por modalidad de terapia sustitutiva.

con mayor número de referencias fue Jalisco, seguida del Estado de México, Puebla, Ciudad de México y Coahuila (Figura 3). Se observó un incremento de la referencia de pacientes en todas las modalidades dialíticas (Figura 4). El porcentaje de referencias fue de 5 y $2.3 \%$ de pacientes trasplantados (Figura 5).

\section{DISCUSIÓN}

Al tercer semestre de 2019, el CENATRA informó que en México existían 549 establecimientos vigentes au- torizados para una o más de las funciones siguientes: 419 con licencia para procuración de órganos, 412 para trasplante y 59 para banco de órganos. Respecto a los más de 22,568 sujetos registrados en espera de un trasplante, 16,503 son de riñón y 15,399 pertenecen al Instituto Mexicano del Seguro Social (68.2\%). ${ }^{1}$ El protocolo de estudio en el donador renal incluye una diversidad de estudios bioquímicos, bacteriológicos, virales, inmunológicos y de imagen, que de manera relativa ofrecen cierto grado de complejidad al equipo de salud; sin embargo, una vez que este grupo multidisciplinario adopta esta práctica y los estudios se realizan con mayor frecuencia, se convierten en procedimientos cotidianos, en donde los profesionales de la salud se adaptan, los integran y dominan; de manera que prácticamente no existen obstáculos para realizarlos. ${ }^{5}$ Los laboratorios de histocompatibilidad aún no tienen los recursos necesarios para realizar pruebas cruzadas y human leucocyte antigen (HLA), que deberían dar la pauta inicial para el estudio tanto de donadores como receptores, lo cual sería de gran utilidad para mejorar la donación de manera local, regional y hasta internacional, ${ }^{6,7}$ práctica no aprobada por la Ley General de Salud de México; por lo tanto, no promovida por el IMSS. La supervivencia del injerto renal en los últimos años supera el $95 \%$ al primer año de trasplante y alcanza el $90 \%$ a los cinco años, lo cual es producto de la mejor selección de los donadores y la efectividad de los medicamentos inmunosupresores. ${ }^{8,9}$ La donación

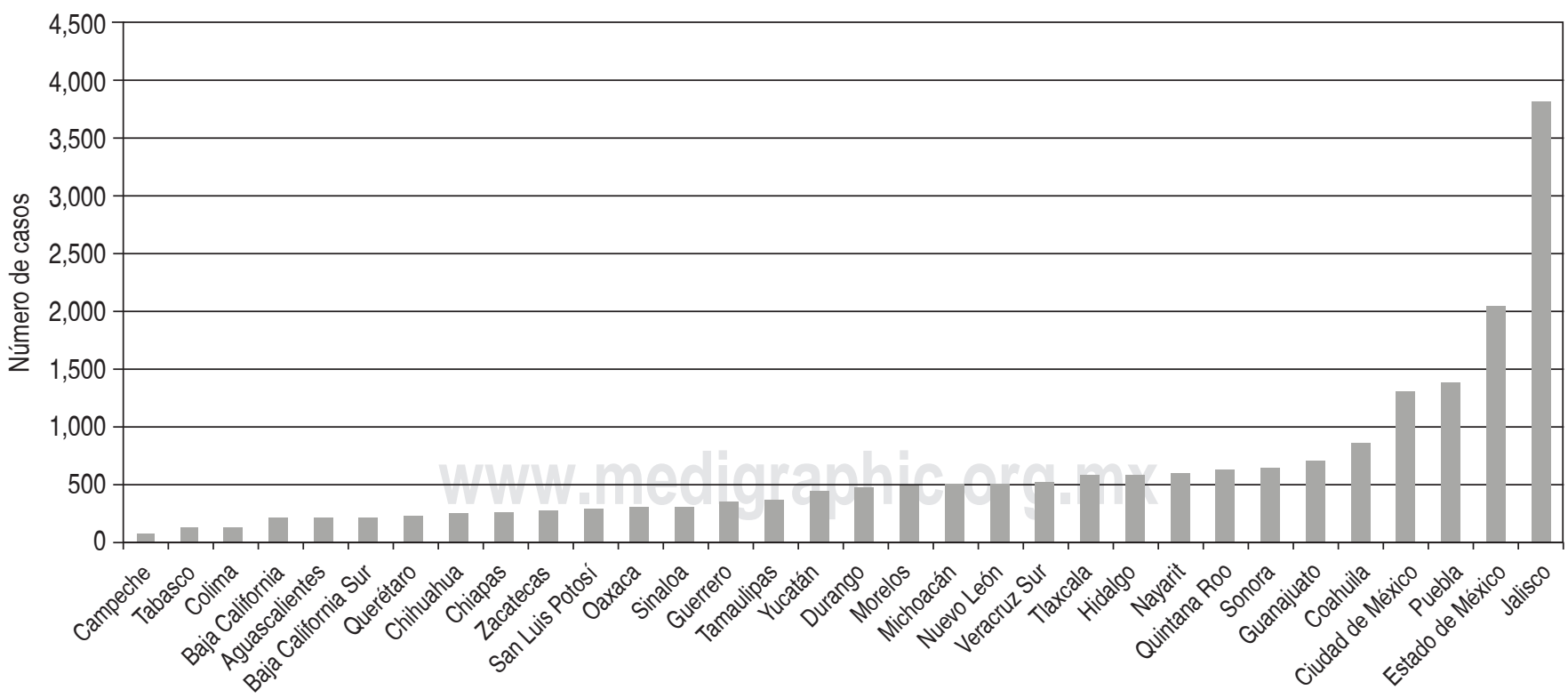

Figura 3: Número de pacientes referidos por localidad geográfica $(\mathrm{N}=19,979)$. 


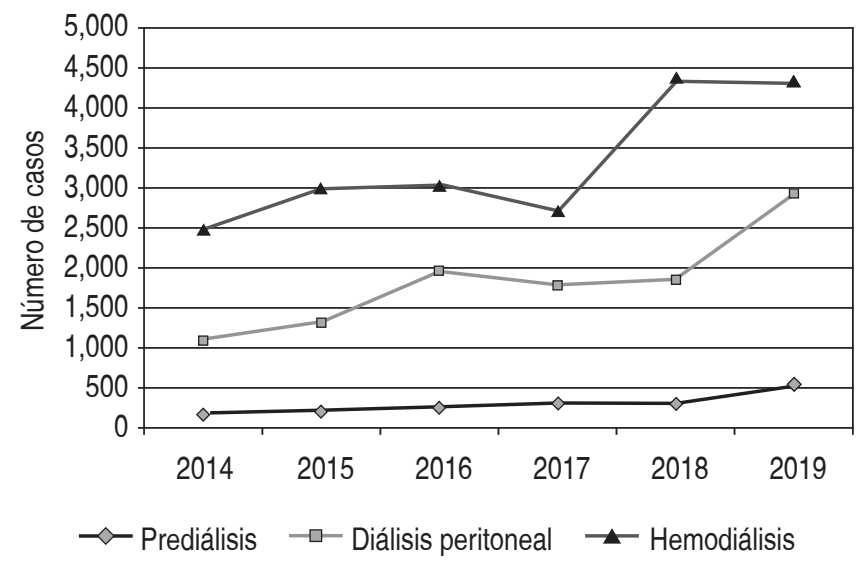

Figura 4: Comportamiento de la referencia por modalidad dialítica.

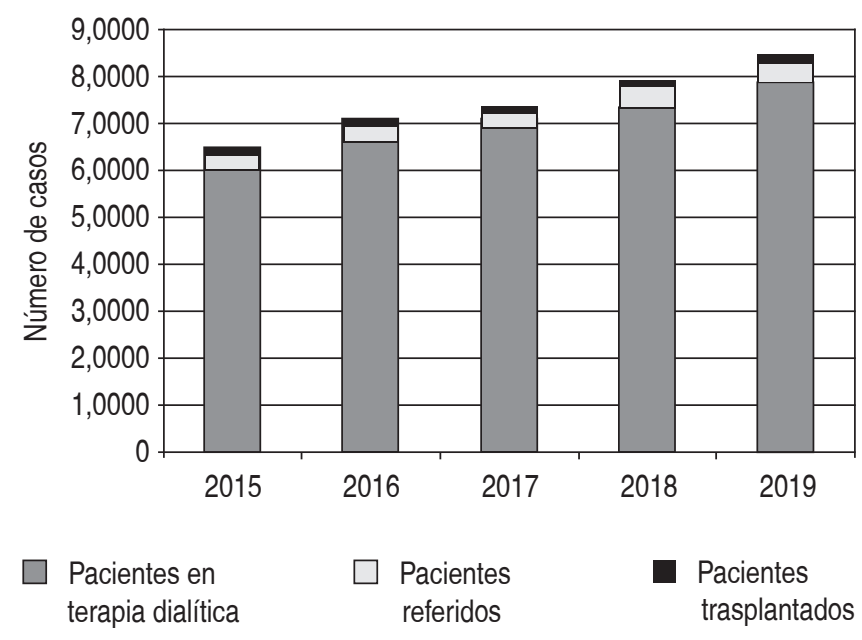

Figura 5: Proporción de pacientes en terapias dialíticas, referidos y trasplantados.

cadavérica continúa constituyendo un reto en México, existe un mayor número de donantes, pero no los suficientes para satisfacer la demanda; esta institución realiza hasta $20 \%$ de trasplantes de origen cadavérico. Un aspecto relevante en la negativa para donar es la creencia religiosa, causas personales del paciente y del entorno familiar; aunque también la procuración de órganos exige personal capacitado para llevar a cabo este proceso hospitalario; ${ }^{10-12}$ al respecto, el IMSS ha implementado la figura de Coordinador Hospitalario de Trasplantes, con resultados alentadores. ${ }^{13}$ México debe continuar realizando estrategias para mejorar la donación de órganos. Es de gran relevancia comentar lo relacionado con el ámbito médico, en donde la falta de incentivos al equipo extractor y trasplantador re- dunda en la falta de cooperación para esta actividad. Extender oportunidades con participar en el intercambio institucional, interinstitucional e internacional de órganos, que además contribuye a la mejora, y ofrece la posibilidad de una compatibilidad perfecta entre donante y receptor al impulsar en mayor medida la donación de órganos de cadáveres, facilitar la difusión de los beneficios del trasplante renal a través de los medios de comunicación masiva, actualizar la legislación en materia de donación renal, impulsar la creación de registros institucionales e interinstitucionales, difundir la información actualizada estandarizada a las unidades médicas involucradas en este proceso.

\section{CONCLUSIONES}

La referencia presenta un incremento continuo y los pacientes de prediálisis cada día se refieren con mayor frecuencia. La demanda de cirugía de trasplante renal supera la demanda que la institución presenta y ofrece una gran área de oportunidad para favorecer a un mayor número de pacientes.

Financiamiento: Para la realización de este trabajo no se obtuvo financiamiento de ningún tipo.

Aspectos éticos: Esta investigación no exploró nombres 0 datos personales de los pacientes, así como tampoco de los médicos referidores, la información se mantiene bajo confidencialidad, por lo que su difusión no representa riesgo para los participantes ni los investigadores. ORCID: https://orcid.org/0000-0002-2596-4581

\section{REFERENCIAS}

1. Estado actual de receptores, donación y trasplantes. 3er. trimestre 2019. Centro Nacional de Trasplantes. Secretaría de Salud. Gobierno de México. 2019. Disponible en: https://www. gob.mx/cms/uploads/attachment/file/500964/3erTrimestre2019. pdf Consultado 13 de enero de 2020.

2. Solís-Vargas E, Evangelista-Carrillo LA, Puentes-Camacho A, Rojas-Campos E, Andrade-Sierra J, Gómez-Navarro B et al. Epidemiological characteristics of the largest kidney transplant program in Mexico: Western National Medical Center, Mexican Institute of Social Security. Transplant Proc. 2016; 48 (6): 19992005.

3. Méndez DA, Ignorosa Luna M, Pérez AG, Rivera RF, González IJ, Dávila TJ. Estado actual de las terapias sustitutivas de la función renal en el Instituto Mexicano del Seguro Social. Rev Med Inst Mex Seguro Soc. 2016; 54 (5): 588-593.

4. Knoll G, Cockfield S, Blydt-Hansen T, Baran D, Kiberd B, Landsberg $D$ et al. For the Kidney Transplant Working Group of the Canadian Society of Transplantation. Canadian Society of Transplantation consensus guidelines on eligibility for kidney transplantation. CMAJ. 2005; 173 (10): 1181-1184. 
5. Lia Y, Lib J, Fub Q, Chenb L, Feib J, Dengb S et al. Kidney transplantation from brain-dead donors: initial experience in China. Transplant Proc. 2016; 48 (8): 2592-2595.

6. Bahena CL. Trasplante cruzado y ABO incompatible. Cambio en el panorama tradicional de trasplante renal en México. Rev Sanid Milit Mex. 2018; 72 (2): 133-140.

7. Terner M, Redding N, Wu J. Increasing rates of kidney failure care in Canada strains demand for kidney donors. Healthc $Q$. 2016; 19 (3): 10-12.

8. Kroth LV, Barreiro FF, Saitovitch D, Traesel MA, D'Avila DO, Poli-de-Figueiredo CE. Kidney transplantation at a Southern Brazilian university hospital: a 35-year practice review. Transplant Proc. 2016; 48 (7): 2272-2275.

9. Kramer A, Stel VS, Abad Diez JM et al. Renal replacement therapy in Europe-a summary of the 2010 ERA-EDTA Registry Annual Report. Clin Kidney J. 2013; 6 (1): 105-115.

10. Molnar MZ, Mehrotra R, Duong U, Bunnapradist S, Lukowsky LR, Krishnan $M$ et al. Dialysis modality and outcomes in kidney transplant recipients. Clin J Am Soc Nephrol. 2012; 7: 332-341.
11. Lim HJ, Jambaldorj E, Lee Y, Kang SS, Koo TY, Ahn C et al. Increasing use of the expanded criteria for living kidney donation and good outcomes of living kidney donors in Korea. Transplant Proc. 2016; 48 (7): 2407-2411.

12. Nemes B, Fedor R, Kanyári Z, Lösey L, Juhász F, Kovács DÁ et al. First outcomes, since being full member of Eurotransplant. A single center experience of cadaveric kidney transplantation. Orv Hetil. 2016; 157 (24): 925-937.

13. Boletín Estadístico Informativo del Centro Nacional de Trasplantes BEI-CENATRA. Secretaría de Salud. Gobierno de México. 2017; Vol. II (2): 49.

Correspondencia:

Antonio Méndez-Durán

Durango Núm. 291, piso 12,

Col. Roma Norte, 06700,

Alcaldía Cuauhtémoc,

Ciudad de México, México.

Tel: 57261700 , ext. 14634

E-mail: antonio.mendezd@imss.gob.mx 